\title{
Energy Management in Harvesting Enabled Sensing Nodes: Prediction and Control
}

\author{
Nouman Ashraf ${ }^{1}$, Muhammad Faizan ${ }^{2}$, Waqar Asif ${ }^{3}$, Hassaan Khaliq Qureshi ${ }^{2}$, Adnan Iqbal ${ }^{4}$, and Marios Lestas ${ }^{1}$ \\ ${ }^{1}$ Department of Electrical Engineering, Frederick University, Nicosia, Cyprus \\ ${ }^{2}$ School of Electrical Engineering and Computer Science, National University of Sciences and Technology, \\ Islamabad, Pakistan \\ ${ }^{3}$ School of Engineering and Mathematical Sciences, City, University of London, UK \\ ${ }^{4}$ Department of Electrical Engineering, Namal College, Mianwali, Pakistan \\ ${ }^{1}$ Email: nouman.ashraf@stud.frederick.ac.cy, eng.lm@ frederick.ac.cy \\ ${ }^{2}$ Email: 12mseemfaizan@ @eecs.edu.pk, hassaan.khaliq@seecs.edu.pk \\ ${ }^{3}$ Email: waqar.asif@city.ac.uk \\ ${ }^{4}$ Email: adnan.iqbal@namal.edu.pk
}

\begin{abstract}
Energy efficient transmission rate regulation of wireless sensing nodes, is a critical issue when operating in an energy harvesting (EH) enabled environment. In this work, we view the energy management problem as a queue control problem where the objective is to regulate transmission such that the energy level converges to a reference value. We employ a validated non-linear queuing model to derive two non-linear robust throughput controllers. A notable feature of the proposed scheme is its capability of predicting harvest-able energy. The predictions are generated using the proposed Accurate Solar Irradiance prediction Model (ASIM) whose effectiveness in generating accurate both long and short term predictions is demonstrated using real world data. The stability of the proposed controllers is established analytically and the effectiveness of the proposed strategies is demonstrated using simulations conducted on the Network Simulator (NS-3). The proposed policy is successful in guiding the energy level to the reference value, and outperforms the Throughput Optimal (TO) policy in terms of the throughput achieved.
\end{abstract}

Keywords-Energy harvesting, Energy prediction, Internet of Things, Wireless Sensor Networks, Markov chains, Energy management, Non-linear Control

\section{INTRODUCTION}

The number of Internet of Things (IoT) devices is increasing exponentially [1] and the energy demand for IoT related applications thus continues to grow [2]. Energy efficiency and the life span of IoT devices are key challenges to next generation IoT based solutions [3]-[6]. Therefore, energy management in IoT technologies, is a critical issue. Since, wireless sensing nodes are an integral part of IoT technology [7], [8] the problem of energy management in IoT sensing nodes inherits many of the characteristics of the energy management problem in Wireless Sensor Networks (WSNs) [9]-[11]. Thus throughout the paper, the term "node" is used for IoT and WSN nodes interchangeably. The problem was originally challenged by the limited energy storage capabilities and the independent operation of IoT nodes in remote, often hazardous environments [12]. New challenges and design options later emerged as a result of the integration of the harvesting capability of ambient energy and the emergence of new networking paradigms, as for example in smart cities [2], body sensor networks [13], [14], habitat monitoring [15], volcano monitoring [16], structural monitoring [17], vehicle tracking [18], and more recently nanonetworking [19].

The energy harvesting (EH) capability, whether this is solar, vibration, thermal or energy from radio waves, significantly affects the energy management design [20]. It is well established that the harvesting energy capability penetrates in all layers of network protocol design leading to numerous harvesting aware solutions in a number of network related problems [9], [21]: in topology control [22]-[24], in routing [25], [26], in medium access control [27]-[30] in transmission policies [31], [32], [33], in scheduling based congestion control [34], in data cycling [35], [36] and in admission control [37]. In [22], topology control in the EHWSNs is considered and game theory-based solution is proposed to adaptively adjust the transmission power of each node to utilize the harvested energy efficiently. In [23], the impact of node position on the wireless sensor communication link are studied where sensors are powered by RF EH from an existing wireless system. To enusre the energy neutral operation of nodes, a hierarchical routing protocol is proposed in [24] that groups $\mathrm{EH}$ nodes into a number of clusters. In [25], problem of rout selection in EHWSN is addressed and stability-aware geographic routing scheme is proposed which considers the residual energy, harvested energy, packet reception rate and location information. In [26], the problems of energy consumption and congestion are considered simultaneously. In [27], the design and implementation of the MAC layer of EH enabled wireless embedded systems is considered. An efficient MAC for EH based WSNs is proposed in [28]. A suitable range for the duty cycle is determined in the presence of QoS and network lifetime constraints. Authors in [29] proposed an adaptive energy-efficient algorithm which adapts the MAC parameters of wireless sensors in response to the 
queue occupancy and varying traffic load levels. In [31] and [32], problem of throughput maximization by considering the channel conditions and time varying energy harvesting sources is presented. A geometric water filling (GWF) algorithm is proposed for power allocation with a sum power constraint. Authors in [33] formulated a sum-rate maximization problem of joint resource block and power allocation for the D2D links.

In this work, we examine the problem of determining the transmission policy of wireless sensing nodes, taking into account the constrained energy resources and the EH capability. Several works have appeared in the literature mostly involving stochastic representations of the underlying processes [9], [21]. Stochastic approaches in many cases provide a more realistic representation of the underlying processes and dynamics as energy storage and consumption on wireless nodes is in most cases stochastic in nature. These approaches rely on different formulations of the problem, considering different decision variables of the transmission policy, different design objectives and different characteristics of the stochastic processes involved, whether these are used to represent the energy harvested, the storage devices, the data queueing disciplines or the communications channel. Decision variables include the wake up schedule of the sensing nodes [36], [38], the transmission probability [39], the transmission mode [13], the energy allocated for transmission [40], and the transmission rate [41]. Different design objectives have also been considered as for example the probability of future energy depletion [13], the likelihood of data being correctly detected [13], the average long term importance of the reported data [39], the detection probability [36], the achieved throughput [28], [31], [40], [42][47] and the packet delivery time or transmission completion time [38], [41], [48], [49]. In addition, memoryless [50] and temporally correlated [36], [39] models have been considered, infinite [51] and finite [39], [49], [52] buffer capacities have been accounted for, some cases in the presence of fading communication channels [41], [31], Gaussian relay channels [46] or additive white Gaussian channels [47]-[49]. Note also that battery imperfections have been considered in [53], non ideal circuit power transmitter has been considered in [47], multi-hop duplex communications have been considered in [54], reinforcement learning techniques have been incorporated in [55] and recently in [56] it has been identified that the transmission scheduling policy does affect the EH model, a process which is naturally modelled by feedback. Significant above works involve stochastic representations of the underlying processes [9]. However, stochastic approaches lead to representations which make analysis and design complex in nature and sometimes intractable. Deterministic approaches, however, are in many cases easier to analyze and lead towards simpler to implement solutions. This thrust has led us for the adoption of deterministic approaches in other problems exhibiting similar characteristics to the energy control problem, as for example the congestion control problem in computer networks. So, in this work we adopt a deterministic model approach, we view the problem as a queue control problem and we demonstrate through simulations that the approach is able to outperform a characteristic stochastic approach, despite the simplicity of the deterministic model. The advantage of considering the problem as a queue control problem in a deterministic model framework, is the availability of off the shelf solutions which have been developed in the context of other problems e.g. the congestion control problem. This constitutes the major contribution of this work which paves the way to adopt algorithms from the rich literature of congestion control algorithms available in the literature.

At the same time, recent research efforts in literature have established that EH aware protocols, can be greatly improved upon availability of energy prediction strategies, which generate predictions of harvested energy to be used for better energy provisioning. In [57], node clustering and routing algorithms are proposed to optimize data transmission, based on future predictions of harvested solar energy. Moreover, in [58] a power management scheme for the throughput maximization problem using energy predictions is proposed for the autonomous mode of device-to-device (D2D) communications. In [59], predicted energy based on Kalman filtering is used to regulate the number of bits sent by the transmitter during a time slot in point-to-point communication between wireless nodes. In [60], power in WSNs is controlled using predictions of wind energy, while adaptive control of the packet transmission period with solar EH prediction is proposed in [61]. Such prediction schemes [62] can lead to better energy management of the available and harvested resources and lead to protocols with improved properties and an effective energy provisioning system.

In this work, we consider the energy management problem in IoT sensing nodes and in particular the problem of regulating the transmission rate in the presence of harvested energy whose levels can be predicted. We adopt a design approach based on control theoretic considerations, which is different from the overwhelming body of existing works in the literature. We view the problem as a queue control problem where the objective is to regulate the transmission and thus the energy leaving the battery in order to ensure that at equilibrium the energy level within the battery converges to a constant reference chosen by the designer. Maintaining this reference value ensures that the battery is not depleted and that some energy is stored for crucial or emergency operations. In particular, we consider a non-linear model of the queuing dynamics first introduced in [63], to derive non-linear robust controllers for serving the energy management policy. A notable feature of the proposed protocol is that it incorporates predictions of the energy to be harvested which are generated using the proposed Accurate Solar Irradiance prediction Model (ASIM) and is based on Markov Chains of increasing order [64]. The stability of the proposed controllers is established analytically and the performance of the combined prediction and control policy is investigated using simulations conducted on the Network Simulator (NS-3). Our simulation experiments indicate that both derived controllers are successful in guiding the energy level to the desired values and that the addition of integral action is beneficial in terms of the throughput achieved. Moreover, a sensitivity analysis is performed to investigate the effect of changing the design parameter $\alpha$ and the sampling period. Finally, the proposed approach is compared against the Throughput Optimal (TO) policy [40] 
which is chosen as the benchmark solution on the basis that it is a good representative of throughput maximization policies which are abundant in the literature. Higher throughput is achieved by the proposed strategy. The current work extends our previous works in [64], [65]. The main new contributions of the paper are the following:

- Extension of the ASIM model to account for both long term and short term prediction and demonstration of its effectiveness.

- Introduction of the proportional and integral controller and demonstration of its effectiveness using both analysis and simulations.

- Extensive performance evaluation that includes sensitivity analysis of the proposed schemes (to highlight the robustness) and a comparative analysis relative to an existing approach, namely the TO policy.

The proposed control based approach has not been adopted before and the significance of this work lies on the demonstrated effectiveness of viewing the energy management problem as a queue control problem which was studied extensively in the context of internet congestion control. This paves the way for adopting the rich literature of queue control algorithms derived in that context to serve the energy management problem in WSNs.

The paper is organized as follows: In section II, we describe and validate the proposed prediction scheme, in section III we formulate the energy management problem as a queue control problem and we derive non-linear controllers whose convergence properties are established analytically, in section IV, we evaluate the performance of the combined prediction and control scheme using simulations and finally in section $\mathrm{V}$ we offer our conclusions and future research directions.

\section{ENERGY PREDICTION}

The proposed energy management scheme incorporates predictions of the energy to be harvested. In the proposed scheme, the predictions are obtained using the ASIM model. In this section, we review background theory on the ASIM model and describe how Markov chains of increasing order are used as a baseline to develop the proposed prediction model. We present ASIM model as a generic model for both long term and short term prediction. Central elements of the proposed model are state dependencies of the underlying Markov chain which are determined using a comprehensive data set from different collection points around the world. The main characteristics of the data set are described together with information on how the model is implemented, tuned and validated.

\section{A. The ASIM Model}

In this subsection, we explain ASIM model which is based on Markov chains of increasing order. At first, state dependencies of first order Markov chains are shown and then we describe how it can be extended to the $K^{\text {th }}$ order Markov chain. A random variable $X_{k}$ at the time instant $k$ is considered which can attain values in the set $\left[x_{1}, x_{2}, \ldots, x_{n}\right]$. The probability that a random variable can attain a state $\left(x_{i}\right)$ at a particular time instant depends only on the attained state at the previous time instant according to the Markov property of the first order Markov chains i.e.

$$
\begin{array}{r}
P\left(X_{k+1}=x_{i} \mid X_{k}=x_{j}, X_{k-1}=x_{l}, \ldots, X_{1}=x_{m}\right) \\
=P\left(X_{k+1}=x_{i} \mid X_{k}=x_{j}\right)=P_{j, i}
\end{array}
$$

where $P_{j, i}$ is the transition probability from the state $x_{j}$ to the state $x_{i}$ depicting transition from a state to any other state. The transition of random process can be represented by the probability $\Pi_{i}(k)=P\left(X_{k}=x_{i}\right)$.

$$
\Pi_{i}(k+1)=\sum_{j=1}^{n} \Pi_{j}(k) P_{j, i} \quad, i \in\{1,2, . ., n\}
$$

Within the ASIM model, the range of attainable irradiance values are divided into $n$ equally sized sets to obtain the states. Each set corresponds to a state whose value is equal to the median of the set. For instance, if the maximum solar irradiance value for a particular location is determined to be less than $1000 \frac{W}{m^{2}}, n=10$ different states can be considered where first state corresponds to the range 0 to $100 \frac{\mathrm{W}}{\mathrm{m}^{2}}$.

We now describe how the first order Markov chain can be extended to a general $K^{t h}$ order Markov chain. For the $K^{t h}$ order Markov chain, the probability of the random process attaining a state depends only on the attained states at the previous $K$ time instants i.e.

$$
\begin{array}{r}
P\left(X_{k+1}=x_{i} \mid X_{k}=x_{j}, X_{k-1}=x_{l}, \ldots, X_{1}=x_{m}\right) \\
=P\left(X_{k+1}=x_{i} \mid X_{k}=x_{j}, X_{k-1}=x_{l}, \ldots\right. \\
\left., X_{k-K+1}=x_{q}\right)=P_{j, l, \ldots, q, i}
\end{array}
$$

Denoting by $\Pi_{j, l, \ldots, q}(k)=P\left(X_{k}=x_{j}, X_{k-1}=\right.$ $\left.x_{l}, \ldots, X_{k-K+1}=x_{q}\right)$, the probability $\Pi_{i}(k)=P\left(X_{k}=x_{i}\right)$ is now evaluated according to:

$$
\begin{array}{r}
\Pi_{i}(k+1)=\sum_{j=1}^{n} \sum_{l=1}^{n} \ldots \sum_{q=1}^{n} \Pi_{j, l, \ldots, q}(k) P_{j, l, \ldots, q, i} \\
, i \in\{1,2, \ldots, n\}
\end{array}
$$

For concluding the model, we consider each $K$-tuple as a state and transitions are only possible if the original state and the $K$ element of the destination state are same. As an example, the state $x_{1} x_{2} \ldots x_{3}^{K}$ can make a transition to any state starting with $x_{2} \ldots x_{3}^{K-1}$, e.g. $x_{2} \ldots x_{3}^{K-1} x_{5}^{K}$, where $P_{1,2, \ldots, 3,5}$ is the probability associated with the transition.

The $K^{t h}$ order Markov chain can be defined by its probability matrix, where the probability matrix can be calculated using equations (3) and (4). If there are $k$ states in a Markov chain then the probability matrix takes the form:

$$
P=\left[\begin{array}{cccc}
P_{1, . .1,1} & P_{1, . ., 1,2} & \cdots & P_{1, . ., 1, k} \\
P_{1, . .2,1} & P_{1, . ., 2,2} & \cdots & P_{1, . ., 2, k} \\
\vdots & \vdots & \vdots & \vdots \\
P_{1, . ., k, 1} & P_{1, . ., k, 2} & \cdots & P_{1, . ., k, k} \\
P_{2, . ., 1,1} & P_{2, . ., 1,2} & \cdots & P_{2, . ., 1, k} \\
P_{2, . .2,1} & P_{2, . ., 2,2} & \cdots & P_{2, . ., 2, k} \\
\vdots & \vdots & \vdots & \vdots \\
P_{k, . ., k, 1} & P_{k, . ., k, 2} & \cdots & P_{k, . ., k, k}
\end{array}\right]
$$




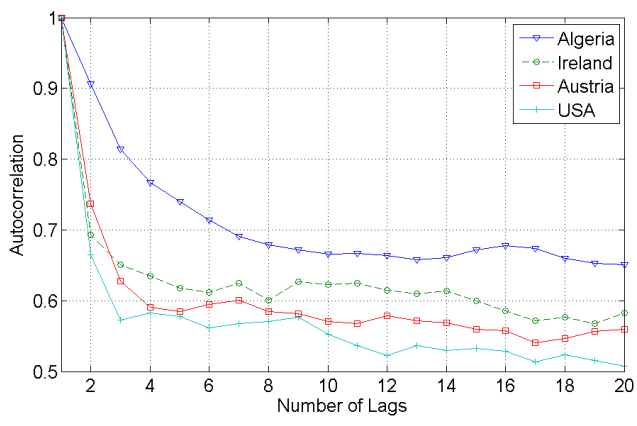

Fig. 1: Autocorrelation for all datasets

The transition probability from the current state $q$ to state $i$ at any time instance $t$ is $P_{j l . q q}$. Therefore, if there are $n$ number of transitions from states $j l . . q$ to state $i$ in the irradiation data, the transition probability $P_{j l . . q i}$ will be $P_{j l . q i}=$ $n_{j l . . q i} / \sum_{i=1}^{k} n_{j l . . q i}$. We now analyse the prediction results of the ASIM model in subsequent subsections.

\section{B. ASIM as a Long-Term Predictor}

In this subsection, we evaluate the performance of the ASIM model for long term predictions using solar radiation data sets. We demonstrate that the predictions obtained from ASIM model show close realization of original radiations. We first evaluate the data set and then demonstrate the results.

1) Long-Term Data Set: The ASIM model is evaluated using real world solar radiation data sets obtained from the World Radiation Data Center (WRDC) [66]. Moreover, long-term data is the accumulated sum of one day (24 hours) radiations. Data sets from four various locations are used namely: Sonnblick (Austria), Bondville (Illinois, USA), Valentia (Ireland), and Tamanrasset (Algeria). The chosen locations are characterized by longer data collection period having variety in daily radiation as shown in Table I. Although in this work the focus has been on solar energy harvesting, the ability of the ASIM model to successfully generate predictions for data sets beyond solar has been demonstrated in [62], where wind energy sets were also considered.

TABLE I: Long-Term Data set information

\begin{tabular}{|l|l|l|l|l|l|}
\hline \multirow{2}{*}{ Location } & \multirow{2}{*}{ Data years } & \multirow{2}{*}{$\begin{array}{l}\text { Mean } \\
\text { Temp. }\end{array}$} & \multicolumn{3}{|c|}{$\begin{array}{l}\text { Daily total Radiation } \\
\left(\mathrm{W} / \mathrm{m}^{2}\right)\end{array}$} \\
\cline { 4 - 6 } & & Max & Min & Avg. \\
\hline Sonnblick, Austria & $1993-2012$ & $-04^{\circ} \mathrm{C}$ & 3828 & 97 & 1450 \\
\hline Bondville, IL, USA & $2003-2012$ & $11^{\circ} \mathrm{C}$ & 3246 & 33 & 1470 \\
\hline Valentia, Ireland & $2003-2012$ & $11.4^{\circ} \mathrm{C}$ & 3165 & 26 & 995 \\
\hline Tamanrasset, Algeria & $2001-2006$ & $21.1^{\circ} \mathrm{C}$ & 3604 & 1968 & 2809 \\
\hline
\end{tabular}

The data sets considered in this study, similar to other real world data sets, have some missing values. It is worth noting, however, that the number of missing values in the selected locations is fairly small $0.05 \%, 0.08 \%, 0.5 \%$ and $0.9 \%$ respectively. In order to fill in the missing values we used the near interpolation technique [67]. Data sets show varying level of dependency among radiation values. We performed

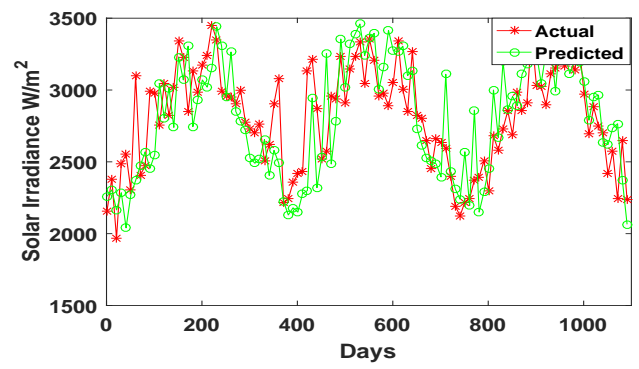

(a) Algeria Data Set

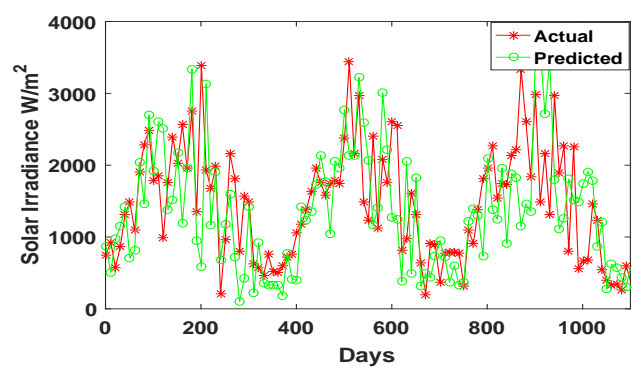

(b) Austria Data Set

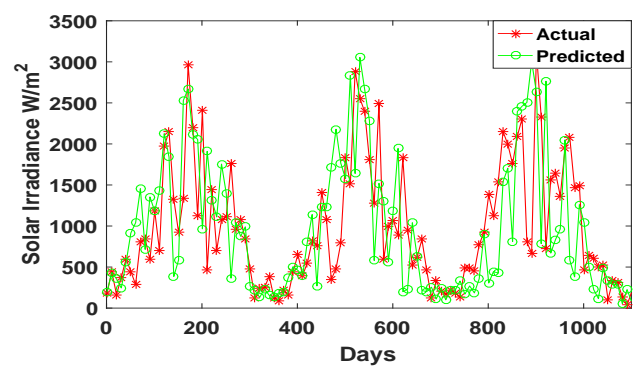

(c) Ireland Data Set

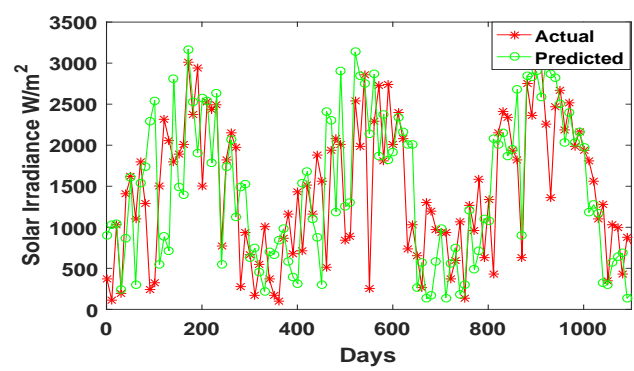

(d) USA Data Set

Fig. 2: Long term data comparison between predicted and the original results

basic autocorrelation analysis to estimate the level of dependency. The results of the autocorrelation analysis are shown in Fig. 1. The Tamanrasset, Algeria trace appears to have longer dependency as it starts converging at about lag 10 and has the highest coefficient values. On the contrary, the Bondville, IL, USA trace starts converging at around lag 6 and has the smallest coefficient values. This suggests that the Tamanrasset data set may require a higher order Markov chain 
to generate predictions of satisfactory accuracy as compared to the Bondville data set. To see the effect of dependent states on accuracy we refer to our previous work [64].

2) Evaluation methodology and Results: To evaluate ASIM model for long term data set, the first half of each trace is used as the training data while the other half is used for evaluating the accuracy of the generated predictions. The considered training data set is divided into bins of size 5 for deriving the states of the Markov chain. Therefore, the first Markov state contains all the solar radiance values between $0-5$ where the largest value of the data set dictates the number of states. For instance, if the largest value is 3165 (Ireland data set), the model will have 633 states. The steady state probability of a transition is calculated by dividing the number of transitions among these states within the considered data set, over all the transitions. The transition probabilities from the attained state obtained during the training phase are used for predicting the next state. This is done by partitioning the $0-1$ interval into regions, where the size of the region is equal to the obtained transition probabilities. The next state is determined by a random number generated following a uniform distribution in the interval $0-1$.

We analyse the accuracy through different methods. First, we use an overlapping graph of actual radiation versus predicted radiations generated by ASIM model to visualize the similarity. We use the reduced form of the ASIM model which incorporates a third order Markov chain with bin size of 5 . It has been shown that such a configuration provides a good trade-off between complexity and performance. Fig. 2 shows the comparison between predicted and real values for the considered locations. The graphs indicate that the predictions provided by ASIM model capture radiations close to the actual radiations. Readers are referred to our recent work [62], where we have compared the performance of of ASIM model with other state of the art prediction models.

We also calculate the Normalized Root Mean Square Error (NRMSE) for each data set. The NRMSE is obtained by dividing Root Mean Square Error (RMSE) with the mean of the observed data in the time interval under consideration. Following standard equation is used to compute NRMSE values $\frac{\sqrt{\frac{\sum_{i=1}^{n}\left\{\frac{A_{i}-P i}{A_{i}}\right\}^{2}}{n}}}{\bar{A}}$, where $A_{i}$ and $P_{i}$ represent actual and predicted values respectively. The error margin is shown in Table III.

\section{The ASIM as a Short-Term Predictor}

In this subsection, we evaluate the ability of the ASIM model to also act as a short term predictor. We collect short term real data sets from WRDC [66]. We consider similar locations to the ones considered in the previous subsection. The short-term data set is the accumulated sum of one hour (60 minutes) radiations. Some basic characteristics of this data are shown in Table II. The evaluation methodology is the same as the one described in the previous section. The change comes from the data set which has hourly granularity instead of daily. The time scale is thus reduced from day to hours. Fig. 3 compares predicted and real data for the considered locations.
The NRMSE is shown in Table III for all the data sets for both short and long term prediction. It may be noted that in Algeria, there are less variations in weather conditions due to less rain and less clouds, thus, more accurate predictions are obtained for Algeria. The results demonstrate the ability of the model to also act as a short term predictor.

Remarks: Reliability of the predictions is dependent on the data sets and application under consideration. For instance, applications where battery storage is small, it is recommended to use short term predictions.

TABLE II: Short-Term Data set information

\begin{tabular}{|l|l|l|l|l|l|}
\hline \multirow{2}{*}{ Location } & \multirow{2}{*}{$\begin{array}{l}\text { Mean } \\
\text { Temp. }\end{array}$} & \multicolumn{3}{|l|}{$\begin{array}{l}\text { Hourly total Radiation } \\
\left(\mathrm{W} / \mathrm{m}^{2}\right)\end{array}$} \\
\cline { 4 - 7 } & & & Max & min & average \\
\hline Sonnblick, Austria & $2014-2015$ & $-04^{\circ} \mathrm{C}$ & 190 & 0 & 25.1 \\
\hline Bondville, IL, USA & $2014-2015$ & $11^{\circ} \mathrm{C}$ & 211 & 0 & 30 \\
\hline Valentia, Ireland & $2014-2015$ & $11.4^{\circ} \mathrm{C}$ & 114 & 0 & 10.5 \\
\hline Tamanrasset, Algeria & $2006-2007$ & $21.1^{\circ} \mathrm{C}$ & 329 & 0 & 78 \\
\hline
\end{tabular}

TABLE III: Normalized RMSE

\begin{tabular}{|l|l|l|}
\hline \multirow{2}{*}{ Location } & \multicolumn{2}{|l|}{ Normalised RMSE } \\
\cline { 2 - 3 } & Long-term & Short-term \\
\hline Sonnblick, Austria & 0.68 & 0.42 \\
\hline Bondville, IL, USA & 1.36 & 0.52 \\
\hline Valentia, Ireland & 0.99 & 0.89 \\
\hline Tamanrasset, Algeria & 0.1 & 0.25 \\
\hline
\end{tabular}

\section{Control Based Energy Management}

In this section, we develop and analyse control based energy management policies for IoT which incorporate energy predictions. The main feature of the proposed scheme, which differentiates it from previous approaches, is that the battery at each node is modelled as a $\mathrm{M} / \mathrm{M} / 1$ queue accommodating energy "packets" and the energy management problem is viewed as a queue control problem. The objective of control problem is to regulate the amount of energy which is made available for packet transmission such that the energy queue level within the battery is maintained at a desired level chosen by the designer. This approach is attractive as it implies that design methodologies which have been used for queue control in the context of congestion control in computer networks can also be applied in this framework. In this work, we adopt this design approach and inspired by [63], we derive nonlinear controllers based on non-linear models of the energy queue. Below, we provide details of the adopted approach and establish the stability properties of the considered controllers analytically.

The battery at each IoT node is modelled as a queue accommodating energy "packets" as shown in Fig. 4. The state of the queue which is the amount of energy stored in the battery is denoted by $x(t)$. The EH capability of the nodes implies an energy input into the battery per unit time which is denoted by $E_{i n}(t)$. Energy is dissipated for network functionality, including transmissions, and the rate of energy dissipation out of the battery is denoted by $E_{\text {out }}(t)$. It is assumed that energy 


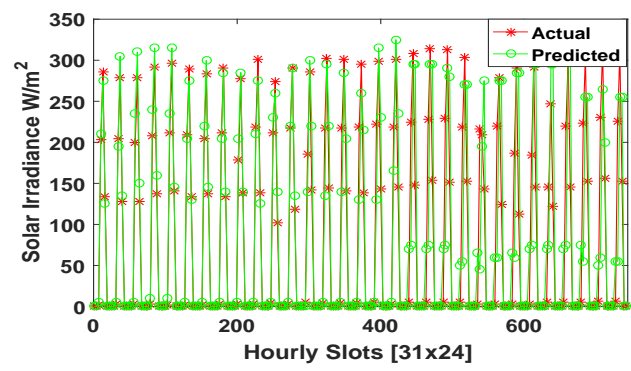

(a) Algeria Data Set

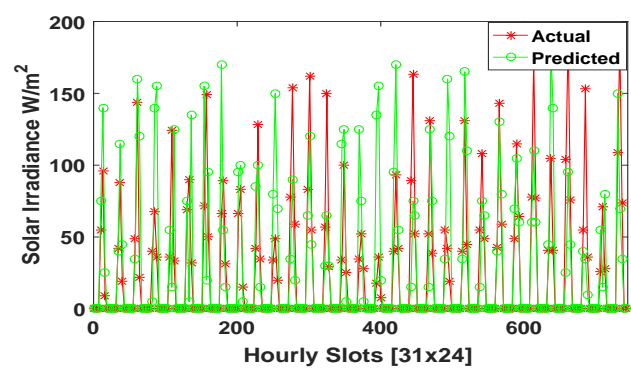

(b) Austria Data Set

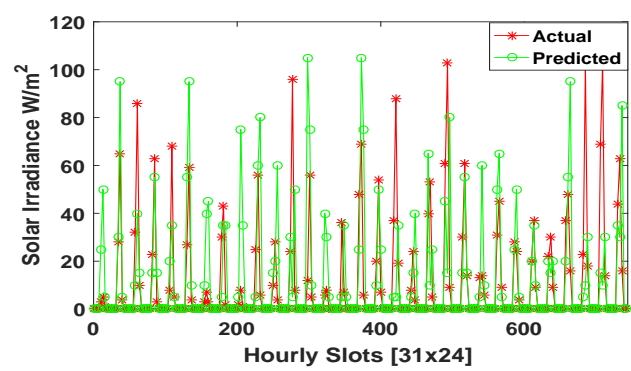

(c) Ireland Data Set

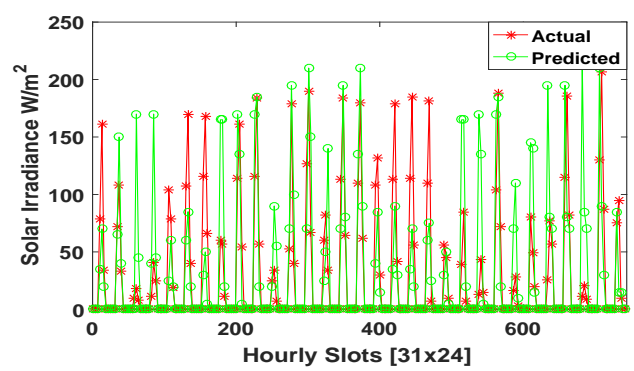

(d) USA Data Set

Fig. 3: Short-term data comparison between predicted and the original results

packet transmission time is proportional to the packet length. It must be noted that, as in many textbooks, the dependence on $t$ of some variables is implied and thus the $(t)$ notation is used interchangeably. Motivated by works [42], [68], [69] indicating that arrival of energy packets to the battery can be modelled as Poisson processes with mean $E_{i n}$, we model the queueing dynamics using a non-linear queueing model. The model was first introduced in [63] and is based on $M / M / 1$ queue, steady

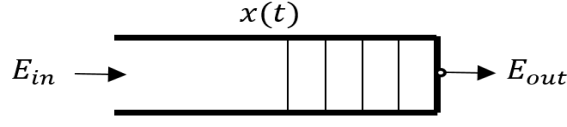

Fig. 4: Queue Model of the Battery

state considerations. The model is shown below:

$\dot{x}(t)=\left\{\begin{array}{l}\max \left(-E_{\text {out }}(t) \frac{x(t)}{1+x(t)}+E_{\text {in }}(t), 0\right), \quad x(t)=0 \\ -E_{\text {out }}(t) \frac{x(t)}{1+x(t)}+E_{\text {in }}(t), \quad 0<x(t)<x_{\max } \\ \min \left(-E_{\text {out }}(t) \frac{x(t)}{1+x(t)}+E_{\text {in }}(t), 0\right), \quad x(t)=x_{\max }\end{array}\right.$

where $x_{\max }$ is the maximum energy that can be stored in battery.

\section{A. Controller}

We adopt this model for developing and analysing our energy management policy. The energy dissipation rate $E_{\text {out }}(t)$, is the control variable, as this can be adjusted by regulating the transmission rate. The control objective is to regulate $E_{\text {out }}(t)$ using predictions of the energy arrival such that the energy stored in the battery tracks a reference energy level denoted by $x_{r e f}$. Maintaining the energy level at $x_{r e f}$ ensures that the battery is not depleted and that some energy is always stored for emergency and crucial operations. The energy predictions, denoted by $\hat{E}_{i n}(t)$, are generated by the ASIM model described in the previous section. Using feedback linearization and robust control concepts, we propose the following control law:

$$
E_{\text {out }}(t)=\max \left[\rho(t) \frac{1+x(t)}{x(t)}\left[\alpha \tilde{x}+\hat{E}_{\text {in }}(t)\right], 0\right]
$$

where $\tilde{x}(t)=x(t)-x_{r e f}$ is the queue error, $\alpha>0$ is a design parameter and $\rho(t)$ is introduced to provide robustness against $x(t)$ attaining small values close to zero and is defined as:

$$
\rho(t)= \begin{cases}0 & \text { if } x(t) \leq 0.01 \\ 1.01 x(t)-0.01 & \text { if } 0.01<x(t) \leq 1 \\ 1 & \text { if } x(t)>1\end{cases}
$$

The proposed control law is used to update the rate $E_{\text {out }}(t)$ with which energy is made available to the wireless sensor node. The update is done every time $T>0$ which is a design parameter and whose effects are discussed in the performance evaluation section. It is assumed that the whole amount of energy which is made available to the sensor node is utilized to send sensed data. So, the more energy is made available to the wireless sensor node, the more data can be sent, and hence higher throughput can be achieved.

Theorem 1: The control law defined by equation (6) guarantees that $x(t)$ is bounded and converges close to $x_{r e f}$ within finite time, with an error that depends on the upper bound of the estimation error $\epsilon$.

Proof: For the proof of Theorem 1, readers are referred to our previous work [65]. 


\section{B. Controller With Integral Term}

In this section, we add integral action and propose another controller for regulating $E_{\text {out }}(t)$, so that energy stored in battery has a fixed value $x_{\text {ref }}$ resulting in availability of some amount of energy every time to keep the nodes alive. Following robust control law based on integral term is proposed.

$$
E_{\text {out }}(t)=\max \left[\rho(t) \frac{1+x(t)}{x(t)}\left[\alpha \tilde{x}(t)+k+\hat{E}_{\text {in }}(t)\right], 0\right]
$$

where $\tilde{x}(t), \alpha$ and $\rho(t)$ are defined earlier in section A. $k$ is the integral term and its derivative is defined as:

$$
\dot{k}=\delta \tilde{x}(t)
$$

with $\delta$ being the design parameter. Moreover, boundary of the neighbourhood $\epsilon$ can be made arbitrarily small by adjusting the design parameter $\delta$.

Theorem 2: The control law described by equation (8) guarantees that $x(t)$ is bounded and converges close to $x_{r e f}$ with time, with an error that depends on the upper bound of the estimation error $\epsilon$.

Proof: Consider the following Lyapunov function:

$$
V(x)=\frac{1}{2} \tilde{x}^{2}(t)+\frac{1}{2} \frac{k^{2}}{\delta}
$$

Differentiating the equation (10) with respect to time yields the following:

$$
\dot{V}(x)=\tilde{x}(t) \dot{\tilde{x}}(t)+\frac{1}{\delta} k \dot{k}
$$

Our objective is to show that $\dot{V}(x) \leq 0$ outside a specific neighbourhood of the equilibrium point $x_{r e f}$. Equation (5) dictates 3 different cases to be considered based on the value of $x(t)$.

Case 1: $\left(0<x(t)<x_{\max }\right)$

For $\left(0<x(t)<x_{\max }\right)$ two sub cases can be further identified. Case 1.1: $E_{\text {out }}(t)>0$

Substituting $E_{\text {out }}(t)$ from equation (8) in (5) yields:

$$
\dot{x}(t)=-\rho(t)\left[\alpha \tilde{x}+\hat{E}_{i n}(t)+k\right]+E_{i n}(t)
$$

Further substituting the value of $\dot{\tilde{x}}(t)$ and $\dot{k}$ from equation (12) and equation (9) respectively, in equation (11) yields:

$$
\begin{array}{r}
\dot{V}(x)=-\rho(t) \tilde{x}^{2}(t) \alpha-k(\rho(t) \tilde{x}(t)-\tilde{x}(t)) \\
-\rho(t) \hat{E}_{i n}(t) \tilde{x}(t)+E_{i n}(t) \tilde{x}(t)
\end{array}
$$

Equation (5) dictates 3 additional sub cases which depend on the value of $x(t)$.

Case 1.1.1: $(x(t)>1)$

Since for $x(t)>1$, according to equation (7) we have $\rho(t)=$ 1. Substituting the latter in equation (13) yields:

$\dot{V}(x)=-\tilde{x}^{2}(t) \alpha-k(\tilde{x}(t)-\tilde{x}(t))-\hat{E}_{i n}(t) \tilde{x}(t)+E_{i n}(t) \tilde{x}(t)$

Let the estimation error be denoted by $\lambda$, where $\left(E_{\text {in }}(t)-\right.$ $\left.\hat{E}_{i n}(t)\right)=\lambda$. It can be assumed that the error is upper bounded by a finite constant $\epsilon$ such that $|\lambda|<\epsilon$. Substituting $\left(E_{\text {in }}(t)-\right.$ $\left.\hat{E}_{i n}(t)\right)=\lambda$ in equation (14) yields:

$$
\dot{V}(x)=-\alpha \tilde{x}^{2}(t)+\lambda \tilde{x}(t)
$$

Completing the square results in the following:

$$
\dot{V}(x)=-\left(\sqrt{\alpha} \tilde{x}(t)-\frac{\lambda}{2 \sqrt{\alpha}}\right)^{2}+\frac{\lambda^{2}}{4 \alpha}
$$

It implies that for $|\tilde{x}| \geq \frac{\epsilon}{\alpha}$, equation (16) leads to $\dot{V}(x) \leq 0$. $\dot{V}(x)$ is negative outside the neighbourhood $\left(|\tilde{x}|<\frac{\lambda}{\alpha}\right)$ i.e for the region $|\tilde{x}| \geq \frac{\lambda}{\alpha}$. With $\epsilon$ being the boundary of the neighbourhood as defined earlier, any solution starting in the neighbourhood will remain therein for all the future times since $\dot{V}(x) \leq 0$ for the region $|\tilde{x}| \geq \frac{\lambda}{\alpha}$. Hence, solutions are uniformly bounded [70], [65]. Further $V(x)$ is monotonically decreasing until the solution enters the neighbourhood. Therefore, we can conclude that the solution is uniformly ultimately bounded [70]. Moreover, the boundary can be made arbitrarily small by adjusting the value of the design parameter $\alpha$.

Case 1.1.2: $(x(t)<0.01)$

For $x(t)<0.01$, equation (7) identifies the value of $\rho(t)=0$. Substituting $\rho(t)=0$ in equation (11) yields:

$$
\dot{V}(x)=E_{i n}(t) \tilde{x}(t)+k \tilde{x}(t)
$$

Since $x(t)<x_{r e f}$, it follows that $\tilde{x}(t)<0$ and in addition $E_{i n}(t)$ is always positive, it follows that $\dot{V}(x) \leq 0$.

Case 1.1.3: $(0.01 \leq x(t) \leq 1)$

Since $0.01 \leq x(t) \leq 1$, thus equation (7) dictates $\rho(t)=$ $1.01 x(t)-0.01$. Substituting the value of $\rho(t)$ in equation (11) yields:

$$
\begin{array}{r}
\dot{V}(x)=-\rho\left[\alpha \tilde{x}^{2}(t)+\tilde{x}(t) E_{i n}(t)\right]+E_{i n}(t) \tilde{x}(t) \\
-k[\rho(t) \tilde{x}(t)-\tilde{x}(t)]
\end{array}
$$

Since $x(t)<x_{\text {ref }}$ it follows that $\tilde{x}(t)<0$ which in turn results in $\dot{V}(x)<0$.

Case 1.2: $E_{\text {out }}(t)=0$

For $E_{\text {out }}(t)=0$ equation (5) gives:

$$
\dot{x}(t)=E_{i n}(t)
$$

Two sub cases can be further identified.

Case 1.2.1: $E_{\text {in }}(t)=0$

Substituting $E_{\text {in }}(t)=0$ in equation (19), $\tilde{\dot{x}}(t)=0$ and $\dot{V}(x)=0$.

Case 1.2.2: $E_{\text {in }}(t)>0$

Since $E_{i n}(t)>0$, It follows that after some time $t_{2} \geq t_{1}$ we will have $\tilde{x}(t) \geq 0$ for $t>t_{2}$ and $\tilde{x}(t)$ will be growing with time $t$. Increasing $\tilde{x}(t)$ implies increasing $x(t)$ which means that there exists a time $t_{3}$ close to the $t_{2}$ i.e $t_{3} \geq t_{2} \geq t_{1}$ such that $E_{\text {out }}(t)$ takes the following value [65]:

$$
E_{\text {out }}(t)=\rho(t) \frac{1+x(t)}{x(t)}\left[\alpha \tilde{x}(t)+k+\hat{E}_{\text {in }}(t)\right]
$$

Moreover, at any instant $E_{\text {out }}(t)=0$ implies that $\tilde{x}(t)<0$, and $\dot{x}(t)=\tilde{\dot{x}}(t)=E_{\text {in }}(t) \geq 0$, which in turn results in $\dot{V}(x) \leq 0[65]$. 
Case 2: $\left(x=x_{\max }\right)$

Since $x(t)=x_{\max }$, considering $x_{\max }>1$, according to equation (7) we have $\rho(t)=1$. Substituting $\rho(t)=1$ in equation (11) results in:

$$
\dot{V}(x)=-\alpha \tilde{x}^{2}(t)+\lambda \tilde{x}(t)
$$

Thus for $|\tilde{x}| \geq \frac{\epsilon}{\alpha}$, it follows that $\dot{V}(x) \leq 0$.

Case 3: $(x=0)$

Since $x=0$, the equation (8) yields $E_{\text {out }}=0$ and substituting the latter in equation (5) yields:

$$
\tilde{\dot{x}}(t)=\max \left[E_{\text {in }}(t), 0\right]
$$

Equation (22) dictates the following 2 additional sub cases to be considered.

Case 3.1: $E_{\text {in }}(t)=0$

Since $E_{\text {in }}(t)=0$, it follows that $\tilde{\dot{x}}(t)=0$ and which in turn results in $\dot{V}(x)=0$.

Case 3.2: $E_{\text {in }}(t)>0$

Since $E_{i n}(t)>0$, it follows the same proof as presented for the Case 1.2.2 and leads to $\dot{V}(x) \leq 0$.

\section{Discrete Time Implementation}

The above presentation of the considered algorithms and their stability analysis was based on continuous time treatment. However, the discrete time version of the proposed control algorithm is more suitable for practical implementation. Below, we present the discrete time version of the controller in equation (6):

$$
E_{\text {out }}(k)=\max \left[\rho(k) \frac{1+x(k)}{x(k)}\left[\alpha \tilde{x}(k)+\hat{E}_{\text {in }}(k)\right], 0\right]
$$

where

$$
\rho(k)= \begin{cases}0 & \text { if } x(k) \leq 0.01 \\ 1.01 x(k)-0.01 & \text { if } 0.01<x(k) \leq 1, \\ 1 & \text { if } x(k)>1\end{cases}
$$

$\tilde{x}(k)=x(k)-x_{r e f}, x_{r e f}$ is the reference queue size and $\alpha$ is a design parameter. Discrete time version of proposed robust controller based on proportional and integral term defined by equation (8) can be represented as:

$$
E_{\text {out }}(k)=\max \left[\rho(k) \frac{1+x(k)}{x(k)}\left[\alpha \tilde{x}(k)+w(k)+\hat{E}_{\text {in }}(k)\right], 0\right]
$$

where $\rho(k)$ is defined earlier in equation (24) and

$$
w(k+1)=\delta \tilde{x}(k) T+w(k), \quad w(0)=0
$$

with $\delta$ being a design parameter and $T$ being the sampling period.

Remarks: The proposed approach consists of two schemes: The Markov based energy prediction scheme and the energy management controller. The implementation complexity of the energy prediction scheme depends on the order of the Markov chain to be utilized and the chosen number of states $n$. The latter is dictated by the range of the available data set and the chosen bin size. The smaller the bin size, the larger is the number of states but the higher is the prediction accuracy. However, it has been demonstrated that reasonably large bin sizes suffice to generate results with good accuracy. The updating matrices are generated offline during the training phase and thus do not contribute to online complexity. The size of these matrices depends on the number of states and the order of the Markov chain. This thus generates the corresponding need for memory requirements. The updating mechanism of the predicted value simply involves selection of a particular row of the relevant matrix and a random number generation. The computational complexity of the energy control scheme can be interpreted from the discrete time version of the proposed controller presented in equation (23) and (24). These algorithms involve limited number of mathematical computations and the implementation complexity is thus reasonable. It must be noted that the complexity is also affected by the sampling period. The smaller the sampling period the higher is the complexity as the computations have to be repeated more frequently.

\section{PERFormance EVAluation}

In the previous section, using analysis, performance bounds were derived for provable controlled behaviour. In this section, we demonstrate using simulations conducted on the NS-3 that the proposed approach achieves the desired behaviour and performance. For this, a network of 100 harvesting enabled wireless sensor nodes is considered where nodes are placed in an area of $1500 \times 1500 \mathrm{~m}^{2}$ following a uniform random distribution. 802.11 transceivers are used with the transmission power value set to $7.5 \mathrm{dbm}$. We considered DCF (Distributed Coordination Function) mode of 802.11 which allows the devices to communicate in an adhoc fashion and therefore forms a kind of WSN. The most commonly used Friss loss propagation model is adopted. In the considered scenario, a randomly selected set of 20 source/sink pairs initiate the communication in the network by transmitting packets with a rate of $2.048 \mathrm{~Kb} / \mathrm{s}$ each. Each packet size is restricted to 64 byte. The packets are relayed between nodes based on the OLSR (Optimized Link State Routing) protocol. All measurements are recorded after $100 \mathrm{sec}$ which provides sufficient time for the OLSR algorithm to converge to its equilibrium state. The $\mathrm{EH}$ enabled nodes periodically update the EH status based on equation (23) and determine the data transmission rate. The control period is denoted by $T$ and is set to $T=10 \mathrm{sec}$ in the reference scenario. The harvested energy, which is used to update the energy level in the battery, is determined from real data solar irradiance sets obtained in Austria [66]. Each dataset contains solar irradiance for two years (2011 and 2012) having the granularity of one value per 30 minutes. This data is used to generate energy predictions according to short term version of our proposed ASIM model [64], whose performance has been demonstrated in section III of the paper. Multiple simulations are run to establish that the energy consumption for packet transmission is of $0.0025 \mathrm{~J} /$ packet. The battery capacity of a node is assumed to be $1 J$, where battery is initially considered to be fully charged. Initially, the design parameter $\alpha$ which affects the convergence properties of the scheme is set to 1 . Two desired energy levels $x_{\text {ref }}$ are considered indicatively: 


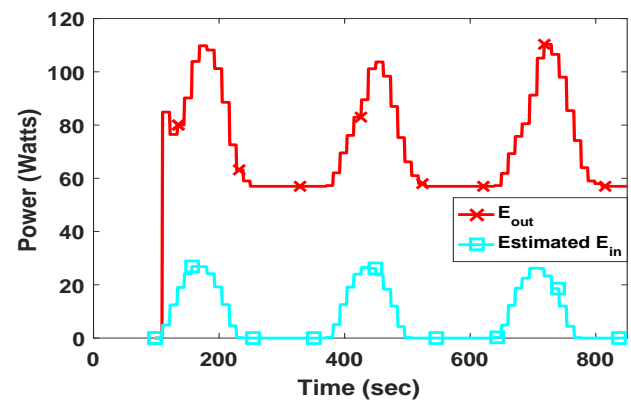

(a) Rate of Input and Output Energy

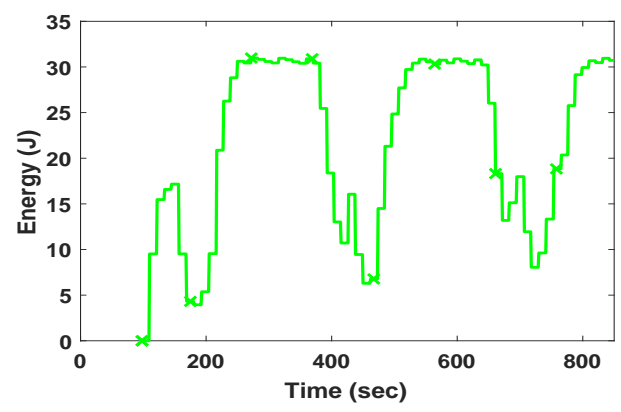

(b) Energy Storage Level

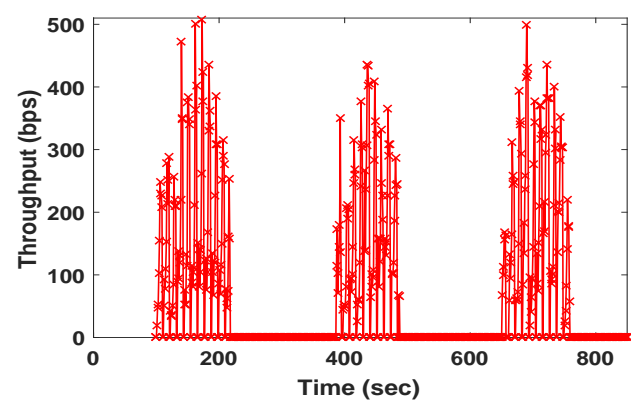

(c) Throughput

Fig. 5: Energy level and Throughput when $x_{r e f}=0.3 \mathrm{~J}$

$0.3 J$ and $0.5 J$. Each node is assumed to be persistent in the sense that it always has data to send.

In the first set of experiments, we set the $x_{r e f}$ value to $0.3 J$ and we report the time evolution of the stored energy of all the nodes in the network and the total network throughput in Fig. $5 b$ and $5 c$ respectively. In order to better understand the observed behaviour, in Fig. 5a we also report the time evolution of the $E_{\text {out }}(t)$ value which indicates the rate of energy leaving the battery to serve the various network operations and the $\hat{E}_{i n}(t)$ value which indicates the predicted energy input rate. It must be noted that the aforementioned values are network values i.e. the sum of the parameter values over all the network nodes. This is done in order to demonstrate the effectiveness of the method for all the network nodes. The corresponding energy levels per node are depicted in the Fig. 6. The first thing to note is that at periods of time for which the system is able to converge to a steady state behaviour, the energy stored in the

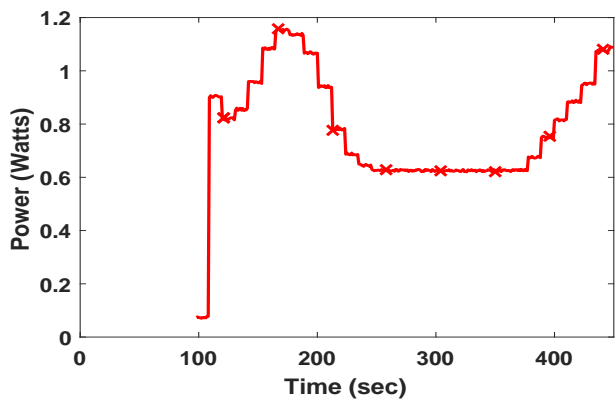

(a) Rate of Output Energy $\left(E_{\text {out }}(t)\right)$ per node

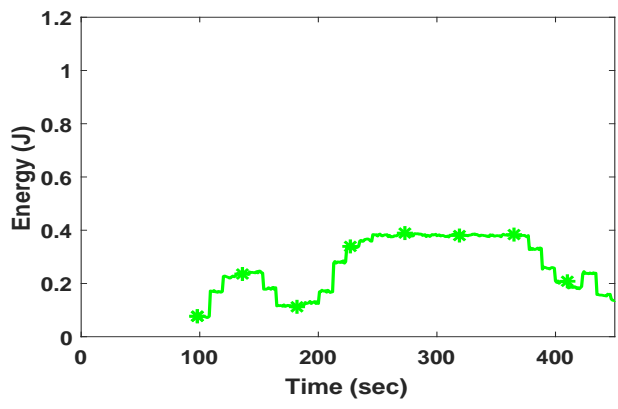

(b) Energy Storage Level per node

Fig. 6: Energy level per node when $x_{r e f}=0.3 J$

battery converges to the desired value of $0.3 \mathrm{~J}$. This indicates that the primal design objective is met. The transient behaviour also reveals some interesting attributes. At the initial and last stages of the simulation, when the predicted harvesting energy increases, indicating future energy availability, the algorithm increases the energy availability for transmission, leading to the reported high throughput values in that period. In those transient periods, the frequent changes in the input signal do not allow the energy level in the battery to converge to the reference level, however, the algorithm is successful in maintaining bounded energy level values which do not allow the depletion of the batteries or the full utilization. Moreover, when the predicted energy input is zero, the algorithm leads to the $E_{\text {out }}$ attaining its lower bound value which implies that no transmissions are allowed leading to the observed zero throughput value. In addition, during this period the battery energy level converges to the posed reference value. Similar behaviour is observed in the case of the reference value being equal to $0.5 \mathrm{~J}$ as depicted in Fig. 7. The proposed algorithm is able to achieve its primal goal which is to converge to an energy level of $0.5 J$ when in steady state.

Feedback control policies, such as the one proposed in this work are known to have good robustness properties i.e. to continue to work effectively in the presence of model uncertainties or disturbances such as for example varying harvesting intakes. However, suitable choice of the reference value can further improve system performance and robustness. For example, at times where extended periods of low harvesting intake can be predicted, the reference value can be set at a relatively high value to account for high processing and communication 


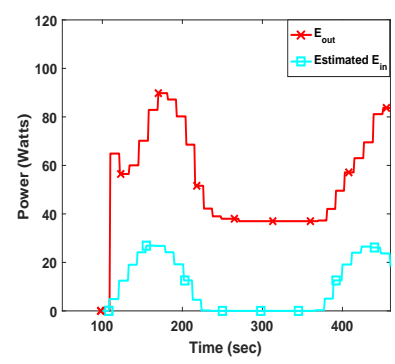

(a) Rate of Input and Output Energy

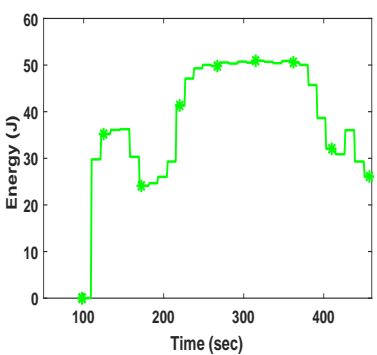

(b) Energy Storage Level

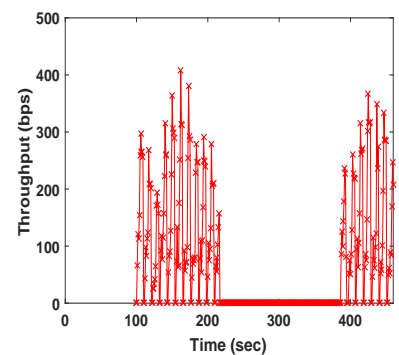

(c) Throughput

Fig. 7: Energy level and Throughput when $x_{r e f}=0.5 \mathrm{~J}$

needs that might arise during that period. Similarly, at periods of expected high energy intake smaller reference values may be selected. As long as the changes in the reference values are not frequent, the algorithm will be able to cope with these changes, with transient properties similar to the ones reported above.

At times of extended low power intake (as for example at night) congestion may occur when the energy management policy does not allow any packet transmission. However, the lack of energy does not leave any alternatives and congestion is preferred rather than total battery depletion. One option that might alleviate the problem to some extent, is to choose high reference values at periods of low harvesting intake. The larger stored energy resources can be used at high congestion situations to alleviate, to some extent the congestion problem.

\section{A. Effect of the sampling period}

Harvesting enabled sensor nodes update their harvesting status after every $T$ seconds and based on equation (23) they determine the data transmission rate. The sampling period $T$ is a major design parameter as it is related to the processing overhead of the scheme and the achieved performance, usually involving a trade-off whose optimal point is in most cases determined using simulations. Control theory dictates that the higher the sampling rate, the better performance is achieved at the expense, however, of higher processing needs as the computations must be performed more frequent. In this subsection, we investigate the effect of changing the sampling period $T$ on the achieved performance. In Fig. 8, we report the time evolution of the battery energy levels and the network throughput when $T$ takes the values $10,30,50$ and 100 sec. As expected, the increase in the sampling period has an

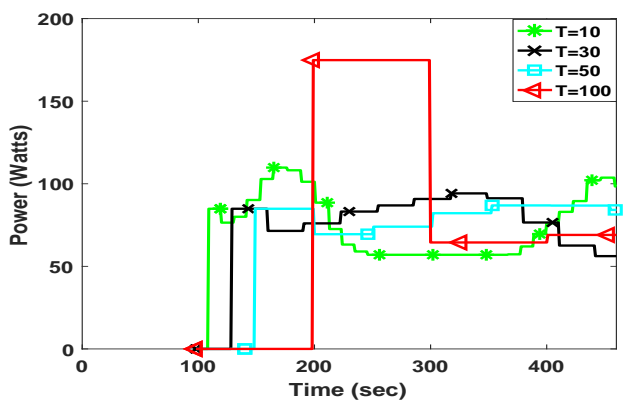

(a) Rate of Output Energy $\left(E_{\text {out }}(t)\right)$

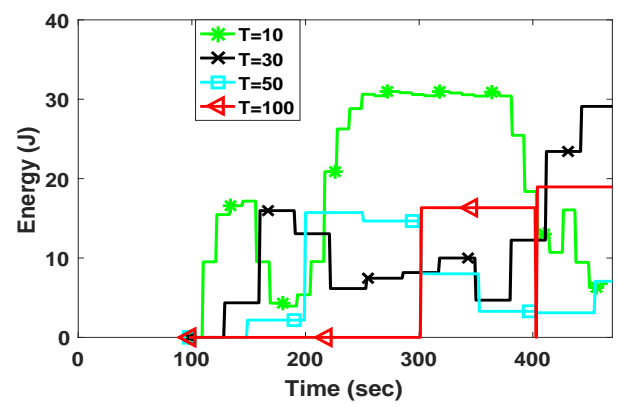

(b) Energy Storage Level

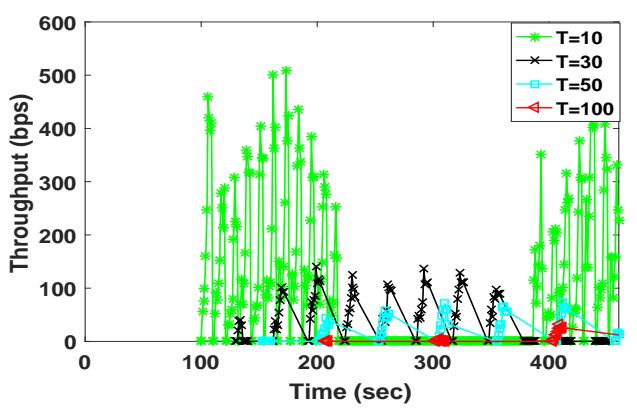

(c) Throughput

Fig. 8: Effects of Sampling Period

adversarial effect on achieved performance. Higher values of the sampling period lead to the algorithm failing to converge to steady state values which in turn results to the energy levels within the battery never achieving the desired reference values. In addition, as the sampling period increases, the reported throughput significantly decreases leading to almost zero values at high sampling periods.

\section{B. Effect of the parameter $\alpha$}

The proposed proportional controller approach, incorporates a single parameter $\alpha$ which needs to be tuned. The parameter $\alpha$ does not have a physical meaning. It is rather a design parameter of the control algorithm. The continuous time analysis performed in the previous sections suggests that the parameter does not affect the stability properties of the system or its steady state behaviour. However, such a parameter is usually introduced to dictate the transient properties of the system, as 
for example the speed of convergence. Due to the simplicity of the adopted model, its effect is difficult to be evaluated using mathematical analysis. In addition, since the implementation is done in a discrete time setting, the effect of the parameter might prove to be different than what is expected from the continuous time analysis. In such situations it is common practice, to evaluate its performance using simulations. This approach has been adopted here as well. In this subsection, we investigate the effect of the parameter $\alpha$ on the achieved performance. We consider the following values of $\alpha: 0.1,0.5$, $1,2,5$ and 10 . We show the reported time evolution of the energy storage, the energy output rate and the throughput in Fig. 9. We observe that the value of $\alpha$ critically affects the system behaviour and that the value of $\alpha=1$ leads to the desired behaviour. As the value of $\alpha$ increases, larger $E_{\text {out }}(t)$ values are generated which leads to the depletion of the available energy resources. This is obviously undesirable. Values of $\alpha$ less than 1 lead to smaller $E_{\text {out }}(t)$ values and thus higher energy storage values at equilibrium. This in turn leads to smaller throughput values.

\section{Effect of adding Integral Action}

The results presented so far refer to the proposed energy queue controller which incorporates feedback linearization principles to apply proportional control action. However, it has been shown in the previous section that the stability properties of the controller are preserved if integral action is also introduced. So, the question which arises is whether the addition of the integral action is beneficial to the observed performance. In this section, we evaluate the performance of the proposed approach when integral action is introduced. The queue reference value is set to $0.3 \mathrm{~J}$ and the total stored energy in the network and the throughput are shown in Fig. 10. It is observed that the controller is successful in guiding the energy queue close to the desired energy level. However, the main advantage is that higher throughput levels are achieved thus demonstrating the beneficial effect of adding the integral action.

\section{Performance Comparison}

The proposed energy management strategy is different in principle from existing strategies in that it poses the problem as a queue control problem where the design objective is to regulate the dissipated energy so that the energy within the battery converges to a reference value dictated by the network operator. The effectiveness of the method has been demonstrated using both analysis and simulations. However, how does the proposed approach compare with existing strategies which are characterized by a different design rationale. Most of the existing schemes pose the objective of regulating the energy dissipation in order to maximize the achieved throughput, or sensing rate in the context of sensor networks. We indicatively choose TO policy [40] as a representative of the aforementioned design rationale and compare its performance against the proposed strategy. The reason that the specific approach was chosen is that the starting point is similar to

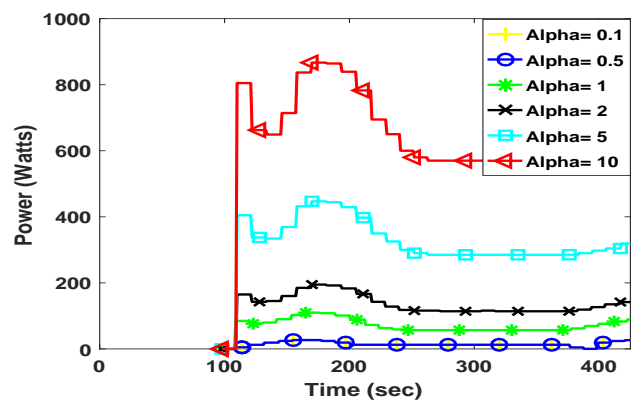

(a) Rate of Output Energy $\left(E_{\text {out }}(t)\right)$

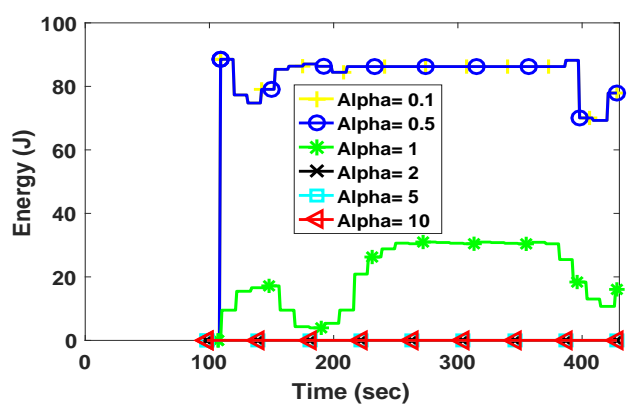

(b) Energy Storage Level

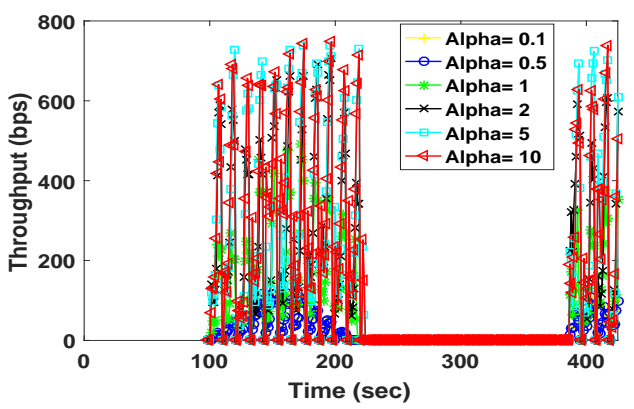

(c) Throughput

Fig. 9: Effects of Changing $\alpha$

our approach, i.e. a queue based representation of the energy storage device. The main difference is that although stochastic models are considered in [40], deterministic models (which are however, based on equilibrium states of underlying stochastic processes) are used in our work. So, the idea is to test two approaches which have the same starting point i.e. queue representation of the energy storage device but they have a different design rationale. The approach in [40] attempts to maximize throughput or minimize delay while our approach is control theory based. It is a comparison between design rationales rather than specific protocols. It is evident from Fig. 11 that the proposed policy is more efficient as at the time instants where more input energy is available, it is able to provide more output energy rate $E_{\text {out }}(t)$ and corresponding higher throughput. At the same time, for the time instants that $E_{i n}(t)$ is small, the proposed scheme is able to retain the battery level to the reference value which can be adjusted by changing the 


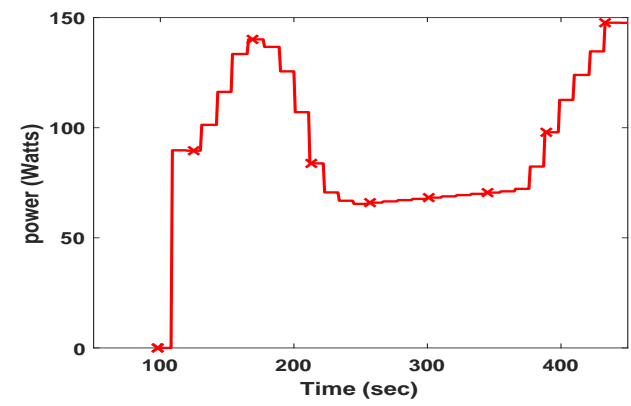

(a) Rate of Output Energy $\left(E_{\text {out }}(t)\right)$

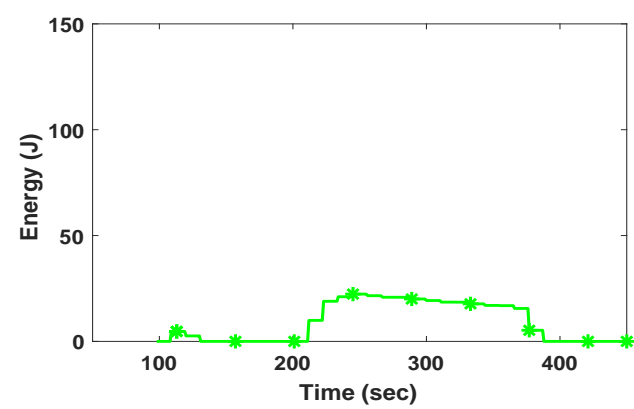

(b) Energy Storage Level

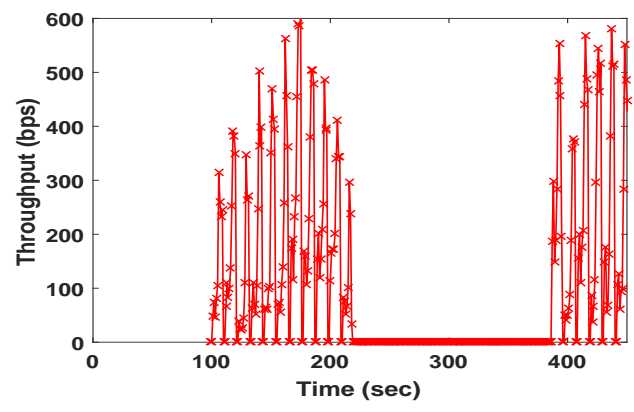

(c) Throughput

Fig. 10: Energy level and Throughput with addition of integral action

value of $x_{r e f}$. In comparison, the TO policy reports less energy for transmission and correspondingly smaller throughput while the energy storage level is higher. Thus, the proposed approach when compared to the TO policy reports a better performance in maintaining the desired energy level simultaneously with adapting the throughput rate according to the available energy levels.

\section{Conclusions}

In this paper, we view the energy management problem in EH enabled IoT sensing nodes, as a queue control problem, where the objective is to regulate transmission so as to guide the battery energy level to a predetermined reference level, and we propose non-linear controllers whose stability properties are established analytically. The proposed strategies utilize predictions of the energy to be harvested which are generated

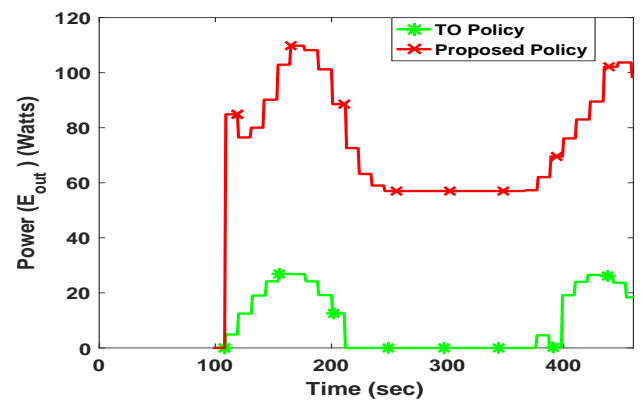

(a) Rate of Output Energy $\left(E_{\text {out }}(t)\right)$

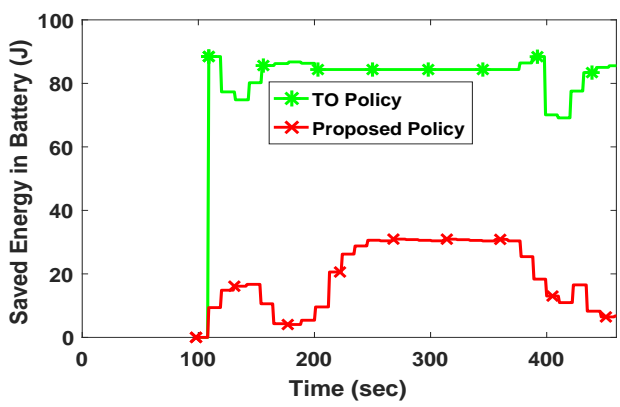

(b) Energy Storage

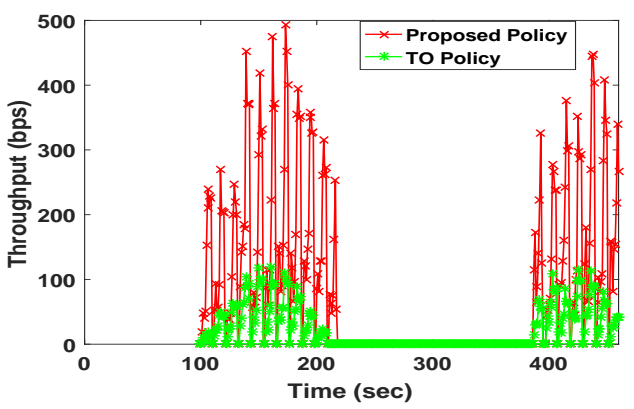

(c) Throughput

Fig. 11: Comparison with TO policy

using the proposed ASIM model. The effectiveness of the proposed method is demonstrated using simulations conducted on the NS-3 simulator. A major contribution of this work is the demonstrated effectiveness of viewing the energy management problem as a queue control problem. So, a natural extension of this work is the consideration of queue control strategies which have been proposed in the context of internet congestion control, in the context of the addressed energy management problem. Another line of future research is the investigation of the combined energy and rate management problem as coupled queue control problems.

\section{REFERENCES}

[1] "Ericsson mobility report," https://www.ericsson.com/en/mobilityreport.

[2] W. Ejaz, M. Naeem, A. Shahid, A. Anpalagan, and M. Jo, "Efficient energy management for the internet of things in smart cities," IEEE Communications Magazine, vol. 55, no. 1, pp. 84-91, 2017. 
[3] N. Kaur and S. K. Sood, "An energy-efficient architecture for the internet of things (iot)," IEEE Systems Journal, vol. 11, no. 2, pp. 796$805,2017$.

[4] R. Arshad, S. Zahoor, M. A. Shah, A. Wahid, and H. Yu, "Green iot: An investigation on energy saving practices for 2020 and beyond," IEEE Access, vol. 5, pp. 15667-15681, 2017.

[5] P. Kolios, G. Ellinas, C. Panayiotou, and M. Polycarpou, "Energy efficient event-based networking for the internet of things," in IEEE 3rd World Forum on Internet of Things (WF-IoT), pp. 1-6, 2016.

[6] J. Iannacci, "Internet of things (iot); internet of everything (ioe); tactile internet; $5 \mathrm{~g}-\mathrm{a}$ (not so evanescent) unifying vision empowered by ehmems (energy harvesting mems) and rf-mems (radio frequency mems)," Sensors and Actuators A: Physical, 2018.

[7] Z. Wadud, N. Javaid, M. A. Khan, N. Alrajeh, M. S. Alabed, and N. Guizani, "Lifetime maximization via hole alleviation in iot enabling heterogeneous wireless sensor networks," Sensors, vol. 17, no. 7, p. 1677, 2017.

[8] M. Shin and I. Joe, "Energy management algorithm for solar-powered energy harvesting wireless sensor node for internet of things," IET Communications, vol. 10, no. 12, pp. 1508-1521, 2016.

[9] S. Ulukus, A. Yener, E. Erkip, O. Simeone, M. Zorzi, P. Grover, and $\mathrm{K}$. Huang, "Energy harvesting wireless communications: A review of recent advances," IEEE Journal on Selected Areas in Communications, vol. 33, no. 3, pp. 360-381, 2015.

[10] G. Anastasi, M. Conti, M. Di Francesco, and A. Passarella, "Energy conservation in wireless sensor networks: A survey," Ad hoc networks, vol. 7, no. 3, pp. 537-568, 2009.

[11] A. Ali, G. A. Shah, and J. Arshad, "Energy efficient techniques for $\mathrm{m} 2 \mathrm{~m}$ communication: A survey," Journal of Network and Computer Applications, vol. 68, pp. 42-55, 2016.

[12] S. Sudevalayam and P. Kulkarni, "Energy harvesting sensor nodes: Survey and implications," IEEE Communications Surveys \& Tutorials, vol. 13, no. 3, pp. 443-461, 2011.

[13] A. Seyedi and B. Sikdar, "Energy efficient transmission strategies for body sensor networks with energy harvesting," IEEE Transactions on Communications, vol. 58, no. 7, pp. 2116-2126, 2010.

[14] F. T. Zuhra, K. B. A. Bakar, A. A. Arain, and M. A. Tunio, "Routing protocols in wireless body sensor networks: A comprehensive survey," Journal of Network and Computer Applications, 2017.

[15] A. Mainwaring, D. Culler, J. Polastre, R. Szewczyk, and J. Anderson, "Wireless sensor networks for habitat monitoring," in Proceedings of the 1st ACM international workshop on Wireless sensor networks and applications, pp. 88-97, 2002.

[16] G. Werner-Allen, K. Lorincz, M. Ruiz, O. Marcillo, J. Johnson, J. Lees, and M. Welsh, "Deploying a wireless sensor network on an active volcano," IEEE internet computing, vol. 10, no. 2, pp. 18-25, 2006.

[17] K. Chebrolu, B. Raman, N. Mishra, P. K. Valiveti, and R. Kumar, "Brimon: a sensor network system for railway bridge monitoring," in Proceedings of the 6th international conference on Mobile systems, applications, and services, pp. 2-14, ACM, 2008.

[18] M. Karpiriski, A. Senart, and V. Cahill, "Sensor networks for smart roads," in Fourth Annual IEEE International Conference on Pervasive Computing and Communications Workshops, pp. 5-pp, 2006.

[19] F. Afsana, M. Asif-Ur-Rahman, M. R. Ahmed, M. Mahmud, and M. S. Kaiser, "An energy conserving routing scheme for wireless body sensor nanonetwork communication," IEEE Access, 2018.

[20] S. Kim, R. Vyas, J. Bito, K. Niotaki, A. Collado, A. Georgiadis, and M. M. Tentzeris, "Ambient rf energy-harvesting technologies for selfsustainable standalone wireless sensor platforms," Proceedings of the IEEE, vol. 102, no. 11, pp. 1649-1666, 2014.

[21] K. S. Adu-Manu, N. Adam, C. Tapparello, H. Ayatollahi, and W. Heinzelman, "Energy-harvesting wireless sensor networks (ehwsns): A review," ACM Transactions on Sensor Networks (TOSN), vol. 14, no. 2, p. 10, 2018.
[22] Q. Tan, W. An, Y. Han, Y. Liu, S. Ci, F.-M. Shao, and H. Tang, "Energy harvesting aware topology control with power adaptation in wireless sensor networks," Ad Hoc Networks, vol. 27, pp. 44-56, 2015.

[23] X. Li, Q. Tang, and C. Sun, "The impact of node position on outage performance of rf energy powered wireless sensor communication links in overlaid deployment scenario," Journal of Network and Computer Applications, vol. 73, pp. 1-11, 2016.

[24] S. Peng, T. Wang, and C. Low, "Energy neutral clustering for energy harvesting wireless sensors networks," Ad Hoc Networks, vol. 28, pp. 116, 2015.

[25] T. D. Hieu, B.-S. Kim, et al., "Stability-aware geographic routing in energy harvesting wireless sensor networks," Sensors, vol. 16, no. 5, p. 696, 2016.

[26] W. Ding, L. Tang, and S. Ji, "Optimizing routing based on congestion control for wireless sensor networks," Wireless Networks, vol. 22, no. 3, pp. 915-925, 2016.

[27] X. Fafoutis, A. Di Mauro, C. Orfanidis, and N. Dragoni, "Energyefficient medium access control for energy harvesting communications," IEEE transactions on consumer electronics, vol. 61, no. 4, pp. 402-410, 2015.

[28] N. Tadayon, S. Khoshroo, E. Askari, H. Wang, and H. Michel, "Power management in smac-based energy-harvesting wireless sensor networks using queuing analysis," Journal of Network and Computer Applications, vol. 36, no. 3, pp. 1008-1017, 2013.

[29] T. D. Nguyen, J. Y. Khan, and D. T. Ngo, "Energy harvested roadside ieee 802.15. 4 wireless sensor networks for iot applications," Ad Hoc Networks, vol. 56, pp. 109-121, 2017.

[30] H. H. R. Sherazi, L. A. Grieco, and G. Boggia, "A comprehensive review on energy harvesting mac protocols in wsns: Challenges and tradeoffs," Ad Hoc Networks, vol. 71, pp. 117-134, 2018.

[31] C. K. Ho and R. Zhang, "Optimal energy allocation for wireless communications with energy harvesting constraints," IEEE Transactions on Signal Processing, vol. 60, no. 9, pp. 4808-4818, 2012.

[32] P. He, L. Zhao, S. Zhou, and Z. Niu, "Recursive waterfilling for wireless links with energy harvesting transmitters," IEEE Transactions on Vehicular Technology, vol. 63, no. 3, pp. 1232-1241, 2014.

[33] S. Gupta, R. Zhang, and L. Hanzo, "Energy harvesting aided device-todevice communication underlaying the cellular downlink," IEEE Access, 2016.

[34] K. Li, W. Ni, L. Duan, M. Abolhasan, and J. Niu, "Wireless power transfer and data collection in wireless sensor networks," IEEE Transactions on Vehicular Technology, 2017.

[35] D. Niyato, E. Hossain, and A. Fallahi, "Sleep and wakeup strategies in solar-powered wireless sensor/mesh networks: Performance analysis and optimization," IEEE Transactions on Mobile Computing, vol. 6, no. 2, pp. 221-236, 2007.

[36] N. Jaggi, K. Kar, and A. Krishnamurthy, "Rechargeable sensor activation under temporally correlated events," Wireless Networks, vol. 15, no. 5, pp. 619-635, 2009.

[37] M. Gatzianas, L. Georgiadis, and L. Tassiulas, "Control of wireless networks with rechargeable batteries [transactions papers]," IEEE Transactions on Wireless Communications, vol. 9, no. 2, 2010.

[38] Y. Wang, H. Chen, X. Wu, and L. Shu, "An energy-efficient sdn based sleep scheduling algorithm for wsns," Journal of Network and Computer Applications, vol. 59, pp. 39-45, 2016.

[39] N. Michelusi, K. Stamatiou, and M. Zorzi, "Transmission policies for energy harvesting sensors with time-correlated energy supply," IEEE Transactions on Communications, vol. 61, no. 7, pp. 2988-3001, 2013.

[40] V. Sharma, U. Mukherji, V. Joseph, and S. Gupta, "Optimal energy management policies for energy harvesting sensor nodes," IEEE Transactions on Wireless Communications, vol. 9, no. 4, 2010.

[41] J. Yang and S. Ulukus, "Optimal packet scheduling in an energy harvesting communication system," IEEE Transactions on Communications, vol. 60 , no. 1 , pp. $220-230,2012$. 
[42] O. Ozel, K. Tutuncuoglu, J. Yang, S. Ulukus, and A. Yener, "Transmission with energy harvesting nodes in fading wireless channels: Optimal policies," IEEE Journal on Selected Areas in Communications, vol. 29, no. 8, pp. 1732-1743, 2011.

[43] K. Tutuncuoglu and A. Yener, "Optimum transmission policies for battery limited energy harvesting nodes," IEEE Transactions on Wireless Communications, vol. 11, no. 3, pp. 1180-1189, 2012.

[44] O. Orhan, D. Gunduz, and E. Erkip, "Throughput maximization for an energy harvesting communication system with processing cost," in IEEE Information Theory Workshop (ITW), pp. 84-88, 2012.

[45] M. Tacca, P. Monti, and A. Fumagalli, "Cooperative and reliable arq protocols for energy harvesting wireless sensor nodes," IEEE Transactions on Wireless Communications, vol. 6, no. 7, 2007.

[46] C. Huang, R. Zhang, and S. Cui, "Throughput maximization for the gaussian relay channel with energy harvesting constraints," IEEE Journal on Selected Areas in Communications, vol. 31, no. 8, pp. 14691479, 2013.

[47] J. Xu and R. Zhang, "Throughput optimal policies for energy harvesting wireless transmitters with non-ideal circuit power," IEEE Journal on Selected Areas in Communications, vol. 32, no. 2, pp. 322-332, 2014.

[48] M. A. Antepli, E. Uysal-Biyikoglu, and H. Erkal, "Optimal packet scheduling on an energy harvesting broadcast link," IEEE Journal on Selected Areas in Communications, vol. 29, no. 8, pp. 1721-1731, 2011.

[49] O. Ozel, J. Yang, and S. Ulukus, "Optimal broadcast scheduling for an energy harvesting rechargeable transmitter with a finite capacity battery," IEEE Transactions on Wireless Communications, vol. 11, no. 6, pp. 2193-2203, 2012.

[50] J. Lei, R. Yates, and L. Greenstein, "A generic model for optimizing single-hop transmission policy of replenishable sensors," IEEE Transactions on Wireless Communications, vol. 8, no. 2, pp. 547-551, 2009.

[51] M. Kashef and A. Ephremides, "Optimal packet scheduling for energy harvesting sources on time varying wireless channels," Journal of Communications and Networks, vol. 14, no. 2, pp. 121-129, 2012.

[52] R. Srivastava and C. E. Koksal, "Basic performance limits and tradeoffs in energy-harvesting sensor nodes with finite data and energy storage," IEEE/ACM Transactions on Networking (TON), vol. 21, no. 4, pp. 1049-1062, 2013.

[53] B. Devillers and D. Gündüz, "A general framework for the optimization of energy harvesting communication systems with battery imperfections," Journal of Communications and Networks, vol. 14, no. 2, pp. 130-139, 2012.

[54] M. Rezaee, M. Mirmohseni, V. Aggarwal, and M. R. Aref, "Optimal transmission policies for multi-hop energy harvesting systems," IEEE Transactions on Green Communications and Networking, 2018.

[55] F. A. Aoudia, M. Gautier, and O. Berder, "Rlman: an energy manager based on reinforcement learning for energy harvesting wireless sensor networks," IEEE Transactions on Green Communications and Networking, vol. 2, no. 2, pp. 408-417, 2018.

[56] Y. Luo, L. Pu, Y. Zhao, W. Wang, and Q. Yang, "Revisiting transmission scheduling in rf energy harvesting wireless communications," arXiv preprint arXiv:1802.09328, 2018.

[57] T. Zou, S. Lin, Q. Feng, and Y. Chen, "Energy-efficient control with harvesting predictions for solar-powered wireless sensor networks," Sensors, vol. 16, no. 1, p. 53, 2016.

[58] U. Saleem, H. K. Qureshi, S. Jangsher, and M. Saleem, “Transmission power management for throughput maximization in harvesting enabled d2d network," in Proc. IEEE Symposium on Computers and Communication, pp. 1078-1083, 2016.

[59] K. Saidi, W. Ajib, and M. Boukadoum, "Adaptive transmitter load size using receiver harvested energy prediction by kalman filter," in IEEE 10th International Symposium on Communication Systems, Networks and Digital Signal Processing (CSNDSP), pp. 1-5, 2016.

[60] A. Jushi, A. Pegatoquet, and T. N. Le, "Wind energy harvesting for autonomous wireless sensor networks," in IEEE Euromicro Conference on Digital System Design (DSD), pp. 301-308, 2016.
[61] K. Kwon, J. Yang, and Y. Yoo, "Adaptive control of the packet transmission period with solar energy harvesting prediction in wireless sensor networks," Sensors, vol. 15, no. 5, pp. 9741-9755, 2015.

[62] H. K. Qureshi, U. Saleem, M. Saleem, A. Pitsillides, M. Lestas, et al., "Harvested energy prediction schemes for wireless sensor networks: Performance evaluation and enhancements," Wireless Communications and Mobile Computing, vol. 2017, 2017.

[63] A. Pitsillides, P. Ioannou, M. Lestas, and L. Rossides, "Adaptive nonlinear congestion controller for a differentiated-services framework," IEEE/ACM Transactions on Networking (TON), vol. 13, no. 1, pp. 94107, 2005.

[64] M. F. Ghuman, A. Iqbal, H. K. Qureshi, and M. Lestas, "Asim: Solar energy availability model for wireless sensor networks," in Proceedings of the 3rd International Workshop on Energy Harvesting \& Energy Neutral Sensing Systems, pp. 21-26, ACM, 2015.

[65] N. Ashraf, W. Asif, H. K. Qureshi, and M. Lestas, "Active energy management for harvesting enabled wireless sensor networks," in IEEE 13th Annual Conference on Wireless On-demand Network Systems and Services (WONS), 2017.

[66] "World radiation data centre," http://wrdc.mgo.rssi.ru/.

[67] S. N. Kersey, "Near-interpolation," Numerische Mathematik, vol. 94, no. 3, pp. 523-540, 2003.

[68] D. W. K. Ng, E. S. Lo, and R. Schober, "Energy-efficient resource allocation in ofdma systems with hybrid energy harvesting base station," IEEE Transactions on Wireless Communications, vol. 12, no. 7, pp. 3412-3427, 2013.

[69] E. De Cuypere, K. De Turck, and D. Fiems, "Stochastic modelling of energy harvesting for low power sensor nodes," in 7th International Conference on Queueing Theory and Network Applications, 2012.

[70] H. Khalil, Nonlinear Systems. Pearson Education, Prentice Hall, 2002. 